\title{
Java for On-line Distributed Monitoring of Heterogeneous Systems and Services
}

\author{
PaOlo Bellavista $^{1}$, Antonio Corradi ${ }^{1}$ And Cesare Stefanelli ${ }^{2}$ \\ ${ }^{1}$ Dipartimento di Elettronica, Informatica e Sistemistica, Università di Bologna, Viale Risorgimento, 2, \\ Bologna, Italy \\ ${ }^{2}$ Dipartimento di Ingegneria, Università di Ferrara, Via Saragat, 1, Ferrara, Italy \\ Email: pbellavista@deis.unibo.it
}

\begin{abstract}
The control and management of Web-based service quality require the extension of the Internet infrastructure with monitoring functions to ascertain dynamically the state of networked resources. We describe the design and implementation of the Monitoring Application Programming Interface (MAPI), a Java-based tool for the on-line monitoring of Internet heterogeneous resources, which provides monitoring indicators at different levels of abstraction. At the application level, it instruments the Java Virtual Machine (JVM) to notify several different types of events triggered during the execution of Java applications, e.g. object allocation and method calls. At the kernel level, MAPI inspects system-specific information generally hidden by the JVM, e.g. CPU usage and incoming network packets, by integrating with Simple Network Management Protocol agents and platform-dependent monitoring modules. MAPI is the core part of a portable tool for distributed monitoring, control and management in the Internet environment. The tool is implemented in terms of mobile agents that move close to the monitored resources to enforce distributed management policies autonomously, with a significant reduction in both reaction time and traffic overhead.
\end{abstract}

\section{INTRODUCTION}

The Internet is becoming an open and global distributed system for service provision to an increasing number of users, interconnected by very different and heterogeneous devices, e.g. PCs, personal digital assistants and even cellular phones [1]. In addition, the enlarging market of multimedia Web services and the competition among service providers stress some innovative service properties that providers, network operators and final customers tend to consider more and more important. One of these properties is the quality of service (QoS), i.e. the ability to control, manage and possibly guarantee negotiated service levels independently of the dynamic conditions of resources in the involved networks and systems [2]. The providers offering services with differentiated QoS levels are interested in accounting users for their real resource consumption and in enforcing the desired billing policies effectively. In the untrusted Internet environment, another crucial service property is security, which permits one to identify and face all forms of misuse and attack, such as denial-of-service obtained by overloading resources to produce unavailability.

In the last few years, several research efforts have introduced ad hoc protocols for QoS management at the network layer [3]. These solutions achieved interesting and effective results when applied to limited networks, but their approach clashes with the best-effort model of the Internet. They require any intermediate router traversed by service packet flows to implement the specific protocol, which is likely to pass through a long process of acceptance and diffusion. As a general consideration, network-layer solutions work at a level of abstraction that makes it difficult to embed some functions which are recognized as fundamental in the state-of-the-art of Internet service provisioning, such as application-specific adaptation and secure billing. Therefore, in order to integrate some forms of QoS differentiation/control with the standard best-effort Internet model, the service infrastructure should be informed of the current usage of heterogeneous resources at runtime. In other words, the service infrastructure should include an on-line monitoring tool able to detect the current condition of network, system and application components during execution. This is necessary to enable dynamic service management via runtime corrective operations.

The monitoring information needed in QoS management covers different abstraction levels, from system conditions at each node (the usage of CPU, memory, bandwidth, etc.), called the kernel state in the following, to the state of application-level service components (the state of a servicespecific daemon process, etc.), sometimes referred to as the application state [4]. In addition, the on-line requirement makes a short response time critical as well as the need to 
reduce the overhead in the observed target, thus forcing one to collect only a restricted set of kernel and application state indicators.

This paper presents the design of a Java-based Monitoring Application Programming Interface (MAPI) for the on-line monitoring of Web services. MAPI overcomes Internet platform heterogeneity and permits one to observe the state of systems/applications during execution. MAPI collects monitoring data at the different levels of abstraction as required. At the application level, it dynamically interacts with the Java Virtual Machine (JVM) to gather detailed information about the execution of Java-based services. At the kernel level, it enables access to system indicators at the monitored target (either Java-based or external to the JVM), such as CPU and memory usage of all active processes.

To overcome the transparency imposed by the JVM, MAPI exploits some recent extensions of the Java technology: the JVM Profiler Interface (JVMPI) [5] and the Java Native Interface (JNI) [6]. In addition, MAPI integrates with external standard monitoring entities, particularly those diffused in the network management domain, i.e. Simple Network Management Protocol (SNMP) agents [7]. JVMPI makes it possible to instrument dynamically the JVM for debugging and monitoring purposes, and MAPI exploits it to collect, filter and analyze application-level events produced by Java applications, e.g. object allocation and method invocation. At the kernel level, MAPI collects systemdependent monitoring data, e.g. CPU usage and incoming network packets, by interrogating SNMP agents that export local monitoring data via their standard management information base (MIB). To also enable the monitoring of hosts without any SNMP agent in execution, MAPI exploits JNI to integrate with platform-dependent monitoring mechanisms, which we have currently implemented for the Windows NT, Solaris and Linux platforms.

We claim that on-line monitoring components play a central role in any distributed infrastructure for QoS-enabled service provision in the Internet environment, to achieve dynamic service adaptation, to enhance global performance, to charge subscribed users for accessed services and to detect possible denial-of-service attacks. This is the reason why we have included the MAPI tool as a core component of a Java-based distributed middleware, called Secure and Open Mobile Agent ${ }^{3}$ (SOMA), for the design, implementation and deployment of Internet services. We have also implemented a MAPI-based distributed monitoring tool in terms of SOMA Mobile Agents that cooperate to enforce distributed management policies. Monitoring agents can migrate close to the networked resources to observe and manage them autonomously on behalf of system administrators, with a significant reduction in both reaction time and traffic overhead.

The paper also reports measurements of the overhead introduced by the local MAPI component and the MAPIbased distributed tool. The monitoring overhead depends

${ }^{3}$ SOMA, MAPI and the MAPI-based distributed monitoring tool are available at http://lia.deis.unibo.it/Research/SOMA/. mainly on the level of detail of monitored indicators and on the time interval for their update. The overhead can be tuned dynamically by service administrators in response to service-/system-specific runtime conditions, and has been shown to be acceptable for most classes of Web services, with good scalability results if compared with traditional SNMP-based centralized solutions.

\section{JAVA TECHNOLOGIES FOR MONITORING}

The Java technology plays a fundamental role in the design, implementation and deployment of Web services over the Internet infrastructure. Apart from Java portability, dynamic class loading and easy integration with the Web, the main motivation of Java diffusion is its virtual machine that hides the local operating system and presents a uniform vision of all available computing resources and middleware facilities.

However, the monitoring perspective requires a complete and low-level visibility of both JVM internals and underlying platforms. At the application level, the MAPI monitoring component exploits JVMPI to acquire visibility of the JVM internal events. At the kernel level, MAPI employs modules external to the JVM, to gather information about platform-dependent resources and nonJava application components. In MAPI, these external modules include both native monitoring mechanisms integrated via JNI and standard monitoring components, i.e. SNMP agents. In the following, the paper briefly describes the JVMPI and JNI technologies, to provide the needed background for the full understanding of the MAPI design and implementation.

\subsection{The JVMPI}

JVMPI is an experimental API of the Java 2 platform, mainly designed to help developers in monitoring Javabased applications during debugging, without imposing any modification in the application code. JVMPI is an interface between the JVM and a dedicated profiler agent, often implemented as a platform-dependent native library for the sake of performance. In one direction, the JVM notifies several VM-internal events to the profiler agent; in the other direction, the profiler agent can enable/disable the notification of specific types of events and can perform some limited management actions on the JVM.

With a finer degree of detail, several JVM conditions trigger JVMPI events: Java thread state change (start, end, when blocking on a locked monitor); beginning/ending of invoked methods; class loading operations; object allocation/deallocation; beginning/ending of the JVM garbage collection. In addition, the profiler agent can use JVMPI to modify dynamically the behavior of monitored applications. Apart from notification enabling/disabling, the agent can intervene on the JVM by invoking a very small set of JVMPI methods: management actions are limited to suspend/resume Java threads and to enable/disable/force the immediate execution of the JVM garbage collector.

The SUN distribution provides a simple implementation of the profiler agent for both Solaris and Windows NT 


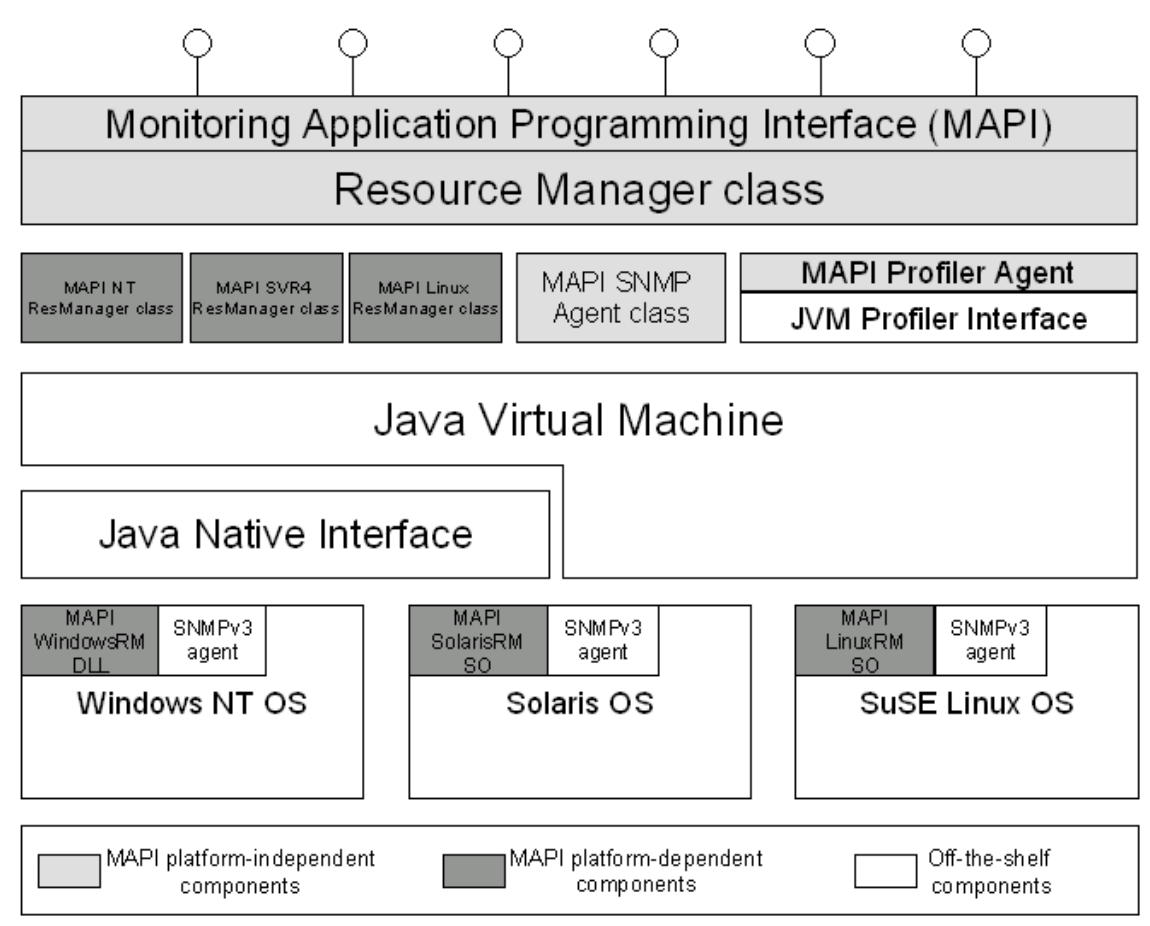

FIGURE 1. The architecture of the Java-based portable MAPI.

operating systems. This agent, called HPROF [5], collects general-purpose events and allows simple static configurations. It is not designed for on-line monitoring, but works mainly as an off-line post-mortem tool for debugging and performance analysis. In fact, it tends to collect a large volume of monitoring data that requires heavy filtering and processing to obtain significant and concise service indicators. For this reason, some researchers have implemented their ad hoc profiler processes to organize HPROF data in immediately readable graphic interfaces $[8,9]$.

\subsection{The JNI}

JNI permits Java threads to invoke native methods, i.e. platform-specific functions typically written in $\mathrm{C} / \mathrm{C}++$, usually available as Dynamic Link Libraries (DLL) in the Windows platform and shared object (SO) libraries under Solaris and Linux.

JNI is a two-way interface. In one direction, a Java program can invoke a native method, by declaring the method with the keyword native and with no body, and by binding to the native method library including the requested function. JNI specifies the details of method invocation: for instance, it rules the parameter marshalling/unmarshalling between the Java invoking thread and the invoked native method. In the other direction, from the native library towards the JVM, JNI allows native methods to interact with their invoking Java framework. JNI permits native code to callback the Java environment and the invoking Java object, to access and modify object values, to call methods and to raise Java exceptions.

With regard to monitoring, MAPI exploits JNI to integrate with native monitoring libraries, in order to obtain the visibility of kernel and application indicators not accessible via JVMPI. For instance, MAPI collects information about process CPU usage by invoking the execution of C-based native libraries that extract the monitoring information differently depending on the monitored target, from either the Windows NT registry or the Solaris / proc directory.

\section{THE MAPI COMPONENT FOR ON-LINE HETEROGENEOUS MONITORING}

When dealing with the best-effort Internet, QoS control and adaptation require the capacity to monitor the level of quality offered by service components. Service components operate in an open and global distributed system, which is intrinsically heterogeneous, and often include legacy elements that cannot be modified/instrumented for monitoring purposes [2, 3, 10]. In addition, the adaptation of Web-service quality requires monitoring information to be available at runtime without imposing any service suspension.

This section presents the MAPI architecture and interface. MAPI permits the on-line monitoring of kernel/application resources independently of possible heterogeneity in their platform implementation. Figure 1 shows that the MAPI interface is implemented by the ResourceManager class, which integrates three different components: the MAPI Profiler Agent, the MAPI SNMP Agent and the MAPI*ResManager (MAPI NT ResManager for Windows, MAPI SVR4 ResManager for Solaris, etc.). MAPI is the core portable component of the distributed monitoring framework described in Section 5.

The MAPI Profiler Agent is able to gather applicationlevel information about the Java environment of the 


\begin{tabular}{|c|c|}
\hline \multicolumn{2}{|c|}{ 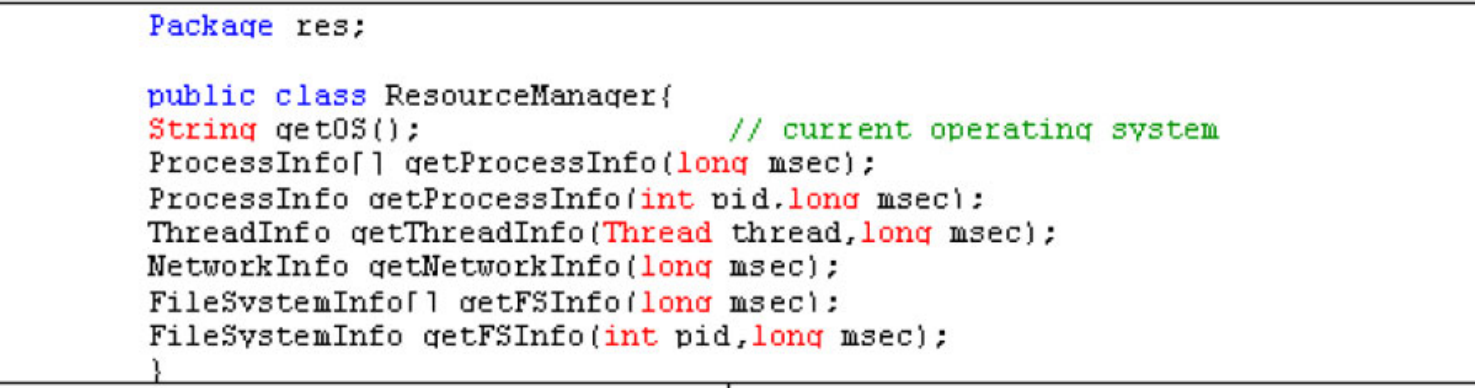 } \\
\hline 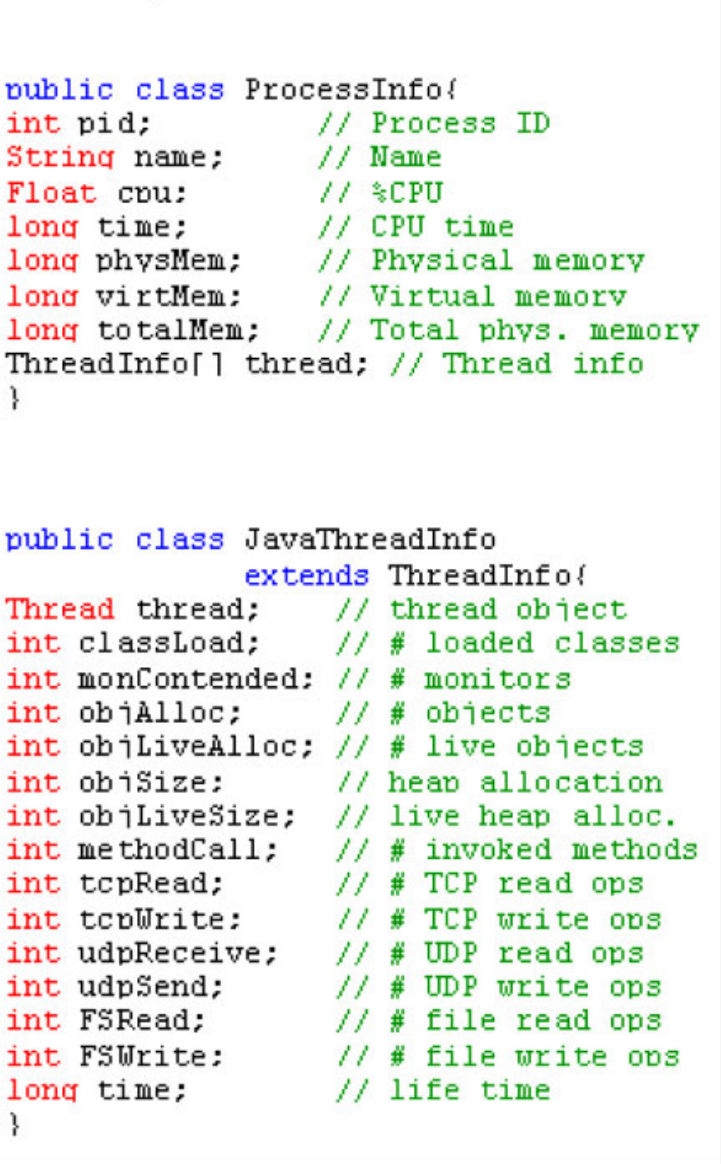 & 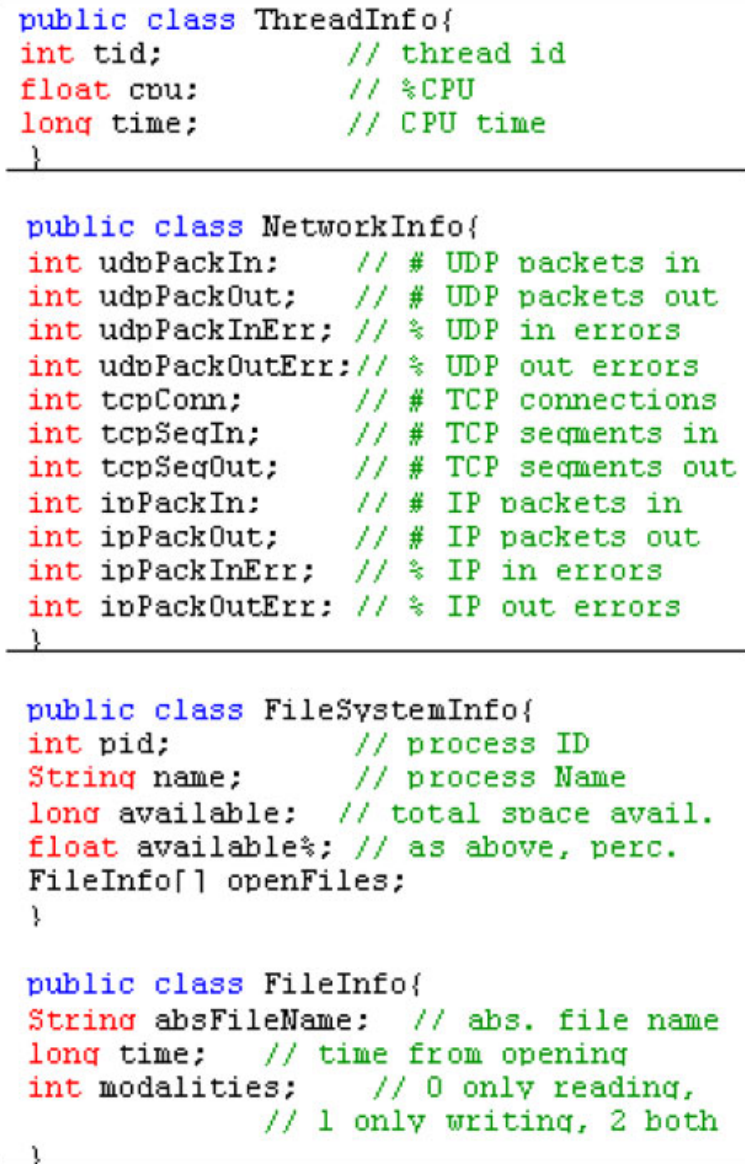 \\
\hline
\end{tabular}

FIGURE 2. The ResourceManager interface.

monitored target. It not only collects JVMPI events but also filters and processes them on-line, to offer concise monitoring indicators during service execution. These JVMPI-based monitoring functions are immediately portable on any host that runs the JVM version 2.

According to the SNMP terminology, the MAPI SNMP Agent acts as an SNMP manager that interrogates the standard SNMP agent available on its local target to obtain kernel-level monitoring data. The MAPI SNMP Agent not only provides a uniform Java interface by wrapping possibly non-Java SNMP agents. It also implements several local optimizations of the SNMP protocol, as described in the following. In addition, it simplifies the configuration of the security parameters needed in SNMPv3, by integrating with the SOMA distributed security infrastructure [11].

To be portable even in the case when the monitored targets do not host the execution of suitable SNMP agents,
ResourceManager exploits the MAPI*ResManager classes to integrate with platform-dependent monitoring functions via JNI. These functions are implemented as native libraries with uniform interfaces for different platforms (MAPI WindowsRM DLL on Microsoft Windows NT 4.0, MAPI SolarisRM SO on SUN Solaris 7 and MAPI LinuxRM SO on SuSE Linux 6.2). ResourceManager achieves portability by sensing dynamically the implementation platform of the current monitored target and by consequently loading at runtime the specific native library.

Figure 2 shows the MAPI set of methods that provide concise monitoring parameters to summarize the current state of the monitored target. MAPI has been designed and implemented to enable service administrators (or autonomous software-based service managers) to obtain kernel/application resource state for on-line service management and adaptation. In this scenario, the overhead is critical 
and monitoring results should be prompt and immediately available to managers (see Section 7). For this reason, MAPI can tune its intrusion to service-specific time constraints: all MAPI methods have a msec invocation parameter that indicates the time interval to update the statistics of collected JVMPI events, to interrogate SNMP agents and to invoke native monitoring libraries.

MAPI methods return either an object or an array of objects of the three classes ProcessInfo, NetworkInfo and FilesystemInfo described in Figure 2. The ProcessInfo object maintains all the data related to the current pid process. Monitored data include the CPU usage (percentage and total time) for any specified process, its allocated memory (physical and virtual) and miscellaneous information on its composing threads. In addition, in the case of JVM threads, MAPI maintains the reference to the Java thread object, its lifetime and the number of loaded classes, used monitors, allocated objects, invoked methods and network and file system operations. For nonJava threads, MAPI provides the thread identifier and the percentage/effective time of CPU usage.

The Network Info class reports aggregated monitoring data about the usage of the communication infrastructure on the target host. Monitored data include the total number of sent/received UDP/IP packets, TCP connections and sent/received segments, the percentage of UDP/IP packets received with errors and the percentage of discarded UDP/IP output packets. These parameters are sufficient to give an overall evaluation of the host traffic conditions and to identify congestion situations.

Finally, the FilesystemInfo class maintains general information about the file system of the target (disk free space and its percentage on total size) and detailed data about currently opened files. In particular, for any active process and for any file opened in the current session, the class returns the opening time and its opening mode (read/write/both/locked).

\section{THE MAPI IMPLEMENTATION}

We have implemented MAPI to be the portable core component of an on-line distributed monitoring tool for the open Internet infrastructure. On the one hand, this imposes the need to achieve complete portability over the most diffused heterogeneous platforms, with the definite implementation constraint of not modifying the standard JVM. On the other hand, Internet openness and dynamicity call for the possibility of monitoring service components without requiring any intervention in either their source code or their executables. The respect of this further implementation constraint makes MAPI a completely original contribution in the Java-based monitoring arena, as detailed further in Section 8.

MAPI has required the design and implementation of several ad hoc modules: (1) the MAPI Profiler Agent for dynamically configurable on-line monitoring of the JVM state; (2) the MAPI SNMP Agent to obtain monitoring data from SNMP agents in execution on the targets; (3) the
MAPI*ResManager and its native libraries (MAPI Windows/Solaris/Linux RM DLL/SO) for uniform data acquisition via heterogeneous platform-dependent mechanisms.

\subsection{The MAPI Profiler Agent}

The SUN JVMPI components significantly constrain the provision of monitoring information. Developers can only specify whether the JVMPI supported events should be notified to the profiler agent, and the specification is coarsegrained, with no possibility of fine selection and dynamic refinement. For instance, a profiler agent can only choose to enable/disable all events related to all Java classes (or objects/methods/monitors), but it can neither focus on the events generated by a specific class nor define user-/ application-specific events.

The only way to obtain more fine-grained indicators is to implement ad hoc profiler agents, as our MAPI Profiler Agent, capable of filtering the events of interest and suitable for composing them in higher level indicators. In addition, the MAPI Profiler Agent gives the possibility of changing the set of notifiable events with no suspension of the monitoring execution, by implementing methods to enable/disable dynamically the event notification related to object allocation/deallocation, method invocation/exit and lock/unlock of Java monitors. Our profiler agent keeps and updates statistics of the monitored events, to provide immediately readable indicators without the need to maintain huge logs of monitoring data. For instance, it traces only the size of the total memory allocated to a Java thread and does not $\log$ the full data related to the execution of any system call for memory allocation.

Figure 3 sketches a piece of the MAPI Profiler Agent code. When a registered event occurs, JVMPI signals an event ev to the profiler that performs event-specific actions. In particular, the figure shows the initializations made when the class Socket Inputstream is loaded. After initializing the internal socketread variable, the profiler can trace any invocation of the method socketRead ( ) by incrementing the stat->tcp_read counter, which maintains the account for the TCP read operations of any Java thread in a specified time interval. These data represent a rough estimation of the incoming network traffic produced by Java service components. If there is the need for more precise information about the traffic due to specific Java threads, ResourceManager can command the profiler to examine dynamically the invocation parameters of the socketRead()/socketWrite() methods. This is possible via the JVMPI-based triggering of JVMPI_EVENT_OBJECT_DUMP of the required objects, at the maximum level of detail (JVMPI_DUMP_LEVEL_2). The MAPI Profiler Agent, of course, behaves differently at default to avoid the excessive overhead of the dynamic generation and processing of object dumps.

\subsection{The MAPI SNMP Agent}

The MAPI SNMP Agent refines and extends the SNMP component included in our MA-based MESIS framework for 


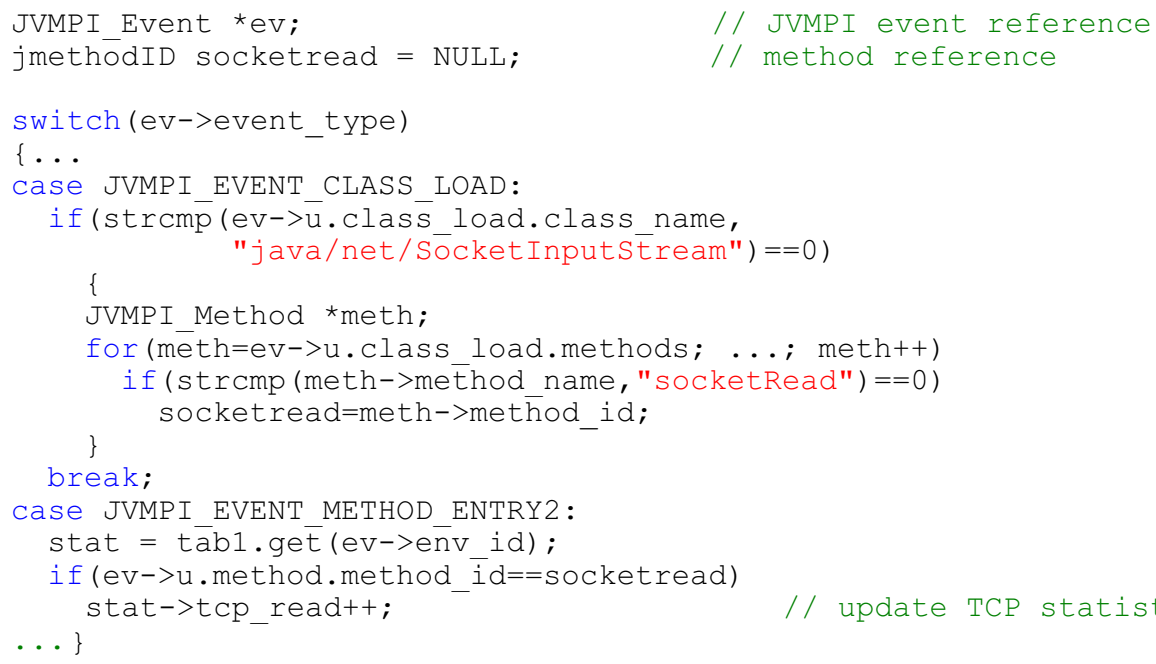

FIGURE 3. Monitoring of the invocation of the socketRead ( ) method in MAPI Profiler Agent.

network and service management [10]. It acts as an SNMP manager that locally interrogates its co-located SNMP agent. The MAPI SNMP Agent is programmed to request monitoring information maintained not only in the standard MIB (monitoring data about network elements and protocols), but also, where supported, in some Host Resources Groups MIB extensions, called Storage, Running Software and Running Software Performance [12]. These groups provide the data about resource usage of processes currently in execution to obtain the MAPI Process Info and FilesystemInfo, while Network Info exploits the standard SNMP MIB.

The MAPI SNMP Agent significantly improves the efficiency of standard client/server SNMP operations, especially when dealing with the network transfer of large chunks of monitoring data. First, it transmits only the changed MAPI indicators to ResourceManager, which maintains old values for the non-received parameters. Most importantly, it locally interrogates its SNMP agent and pre-processes the obtained results to offer concise indicators to possibly remote managers, thus significantly reducing the generated network traffic. In fact, a single MIB variable is usually at a lower level than the MAPI indicators, and an aggregation of multiple variables is required. These aggregations are known as health functions [13]. For instance, the percentage of discarded IP output packets is obtained by combining five MIB variables:

$$
\begin{aligned}
\text { ipPackOutErr }= & {[(\text { ipoutDiscards }} \\
& + \text { ipoutNoRoutes } \\
& + \text { ipFragFails }) * 100] \\
& {[\text { ipOutRequests }} \\
& + \text { ipForwDatagrams }]^{-1}
\end{aligned}
$$

where ipoutDiscards, ipoutNoRoutes and ipFragFails are the number of output IP datagrams discarded (for problems in buffer space, in routing and in fragmentation, respectively), while ipoutRequests and ipForwDatagrams are the total number of IP datagrams transmitted (for locally generated packets and forwarded ones, respectively) [7].

In addition, the MAPI SNMP Agent can perform all the operations needed for the support of mutual authentication in the case of interaction with SNMPv3 agents. It can obtain dynamically the needed security information from the public key infrastructure integrated with the SOMA programming framework $[11,14]$. Finally, it can locally store configuration parameters specific for its SNMP agent (e.g., the supported MIBs), in order to automate the possibly complex phase of initialization of the MAPI tool.

\subsection{The $M A P I *$ ResManager}

To monitor target hosts not supporting either the SNMP agent or the Host Resources MIB extensions, MAPI also integrates native monitoring mechanisms. MAPI native modules extract uniform data by exploiting heterogeneous monitoring mechanisms provided by the target operating systems. The ResourceManager class employs JNI to load the target-specific native library at runtime. We have currently implemented the native monitoring components for Windows NT (MAPI WindowsRM DLL), Solaris (MAPI SolarisRM SO) and Linux (MAPI LinuxRM SO), as depicted in Figure 1.

Figure 4 shows some lines of the MAPI WindowsRM DLL, which accesses kernel and application state indicators maintained in Microsoft system registry keys. In particular, the figure reports the polling of the registry to obtain updated information about the processes in execution. The system call RegQueryValueEx (HKEY_PERFORMANCE_DATA) returns a perfdata reference which is used as the base to access the monitoring information about the process with identifier PID.

For Solaris and Linux platforms, we have implemented native monitoring modules as dynamic SO libraries that mainly exploit the /proc feature. /proc is a virtual directory that exports kernel/application state indicators 


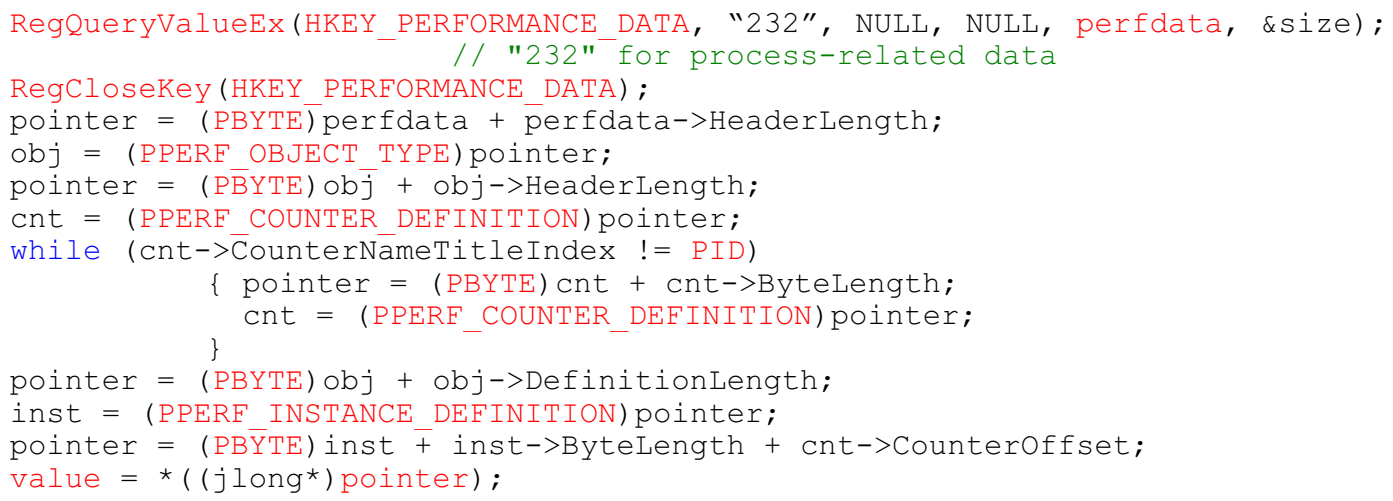

FIGURE 4. Monitoring process information in MAPI WindowsRM DLL.

as a specific sub-tree of the file system. The MAPI SolarisRM/LinuxRM library polls monitoring information about currently executing processes by reading the corresponding files in the / proc directory. For instance, via the ioctl () call it obtains prpsinfo and prusage information, which maintain several data about the identity of a specified process and its CPU usage, respectively. Similarly, SolarisRM/LinuxRM native components extract the descriptors of the open files from the /proc/PID/fd virtual directory, where PID is the identifier of the monitored process, and combine them with the information from the system file table, as in the implementation of the Unix fuser utility. Aggregated information about network usage is obtained by invoking the standard net stat system call [15].

\section{MAPI-BASED DISTRIBUTED MONITORING}

We have designed and implemented a distributed monitoring (DM) tool consisting of mobile agents that use MAPI to monitor each target node. We have adopted the SOMA mobile agent technology to improve the performance in collecting/processing distributed monitoring information and to facilitate the coordination of autonomous monitoring agents. SOMA provides a distributed infrastructure for the design, implementation and support of Web services. It includes middleware facilities for agent mobility, communication, security and interoperability, built on top of the standard portable JVM. Further details about SOMA are presented in $[11,16]$ and are beyond the scope of this paper, which focuses specifically on agents for MAPI-based distributed monitoring.

DM combines two types of monitoring agents: Managers and Explorers. Each Explorer agent is in charge of collecting monitoring data from one set of targets (i.e. target domain), usually belonging to the same network locality. Manager agents command Explorers, combine their monitoring results and are in charge of giving a global view of monitored domains to system administrators. In addition, Managers can delegate some management operations to Explorers that can autonomously control and handle assigned resources according to the enforced management policies, in order to achieve decentralization and scalability [10, 17, 18, 19].
DM permits several organizations, with different hierarchical levels and numbers of Managers/Explorers per target domain. The default choice is one single Manager that autonomously interworks with its Explorers (one Explorer for each administered target domain). In particular, the Manager can either ask Explorers to gather specified monitoring data, with specific alert thresholds and refresh time intervals, or command management operations. In addition, a Manager can create new monitoring Explorers at runtime when in need of controlling new target domains.

Explorer agents migrate to their target hosts to access locally MAPI functions and to collect both kernel and application state indicators. Explorers eventually control dynamic alert thresholds: when thresholds are exceeded, the Explorer either notifies its Manager or autonomously takes local corrective operations. Explorer local decisions significantly reduce the network overhead of distributed monitoring. On the one hand, Explorers can perform autonomous monitoring/management operations locally to the administered resources without the intervention of either Managers or system administrators. On the other hand, they can carry parts of state of their monitoring activities while migrating from one host to another in the target domain. That can permit monitoring/management global decisions based on the state of already visited system parts. SOMA facilitates multi-hop mobility patterns by defining agent itineraries, i.e. sequences of hosts for an agent to visit. Single-hop mobility behavior, suitable for monitoring and controlling resources at one host, is implemented by itineraries that include a single destination. In addition, the SOMA platform supports the caching of agent code on destination targets, thus significantly reducing the migration overhead in the case of usual enforcement of the same management policies [11].

For instance, one Manager agent can be in charge of controlling the storage devices in administered domains. It can delegate one Explorer for each domain to perform cache cleanup of browser directories when the available space becomes lower than a specified threshold. The threshold can state a per-host local constraint or an overall per-domain limit. In the latter case, the Explorer can keep partial results of space availability for the already visited domain targets 
JavaThreadlnfo

File Plot Help

\begin{tabular}{|c|c|c|c|c|c|c|c|c|c|c|c|}
\hline Name & Priorit & Clas. & Obj.. & Memary & Mon. & . Tcp in & Methods & Tc. & Ud. & U. & Life \\
\hline Thread-3 & 6 & 5 & $18 \ldots$ & 79000 & 1 & 0 & 4520 & 0 & 0 & 0 & $0 \mathrm{~s}$ \\
\hline Worker: [Age... & 6 & 29 & 24. & 99232 & 3 & 0 & 12052 & 0 & 0 & 0 & Ds \\
\hline Thread-2 & 6 & 1 & 53 & 16.36 & 3 & 0 & 671 & 0 & 0 & 0 & $0 \mathrm{~s}$ \\
\hline
\end{tabular}

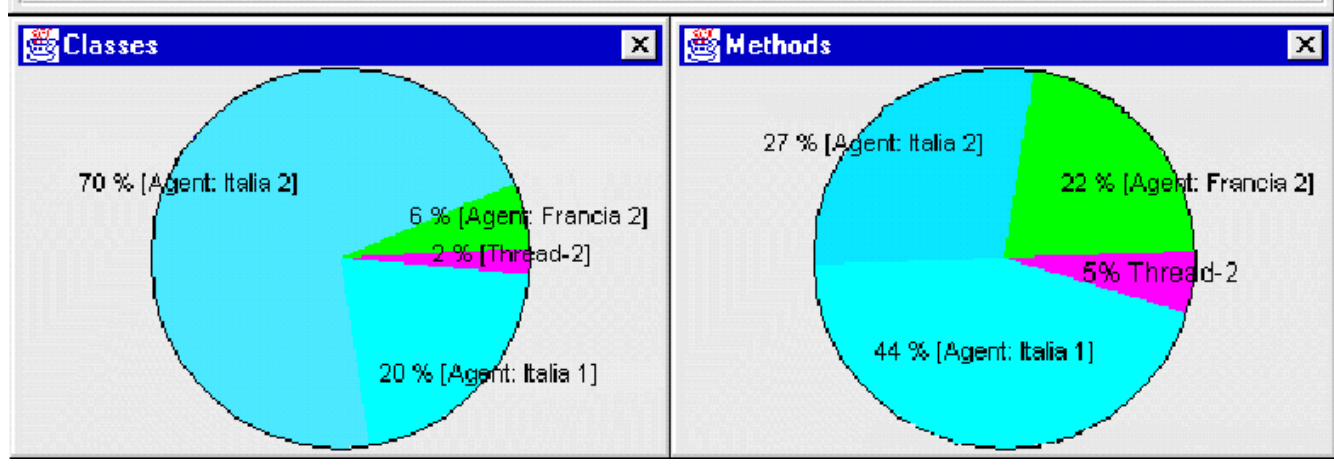

FIGURE 5. Manager GUIs with JavaThreadInfo for a target domain.

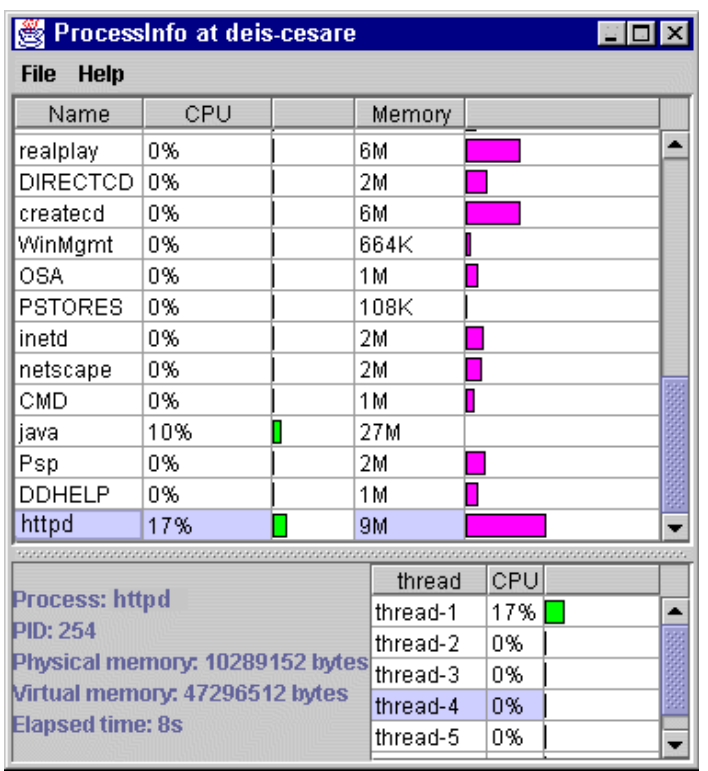

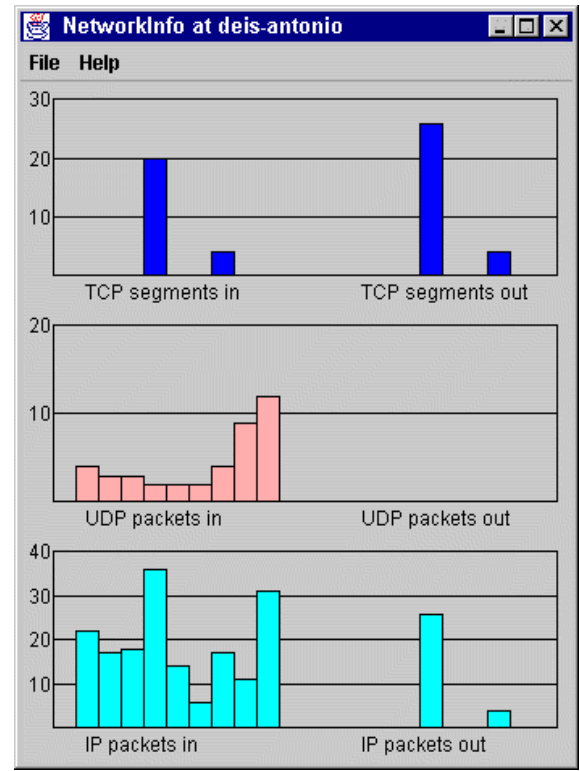

FIGURE 6. Manager GUIs with Process Info and Network Info of two Windows NT targets.

and can suspend migrations when the partial results already satisfy the required threshold.

Explorers can invoke the MAPI Profiler Agent functions to control and manage local Java threads. In particular, they can modify the priority of running threads and can force thread suspension/termination. These functions help in controlling the execution of Java applications and to make possible the limitation of their resource consumption at runtime, as described in the following section. Let us observe that the specification and implementation of the JVM itself limits strictly the control actions feasible with the MAPI Profiler Agent. Additional control functions are recognized as being crucial, especially in the emerging market of Java-enabled embedded devices, and have recently stimulated SUN research work to provide separated execution environments for different Java thread groups (task isolation), to permit independent control [20].

The DM overhead can be tuned at runtime by modifying different monitoring parameters. It is possible to change dynamically both the Explorers visit time interval to target domains and the refresh time interval for MAPI modules. In addition, the Manager can command Explorers to invoke the MAPI Profiler Agent methods to enable/disable the notification of specific kinds of events, thus adapting 
dynamically the collection of monitoring data to the enforced management policy. Section 7 presents the experimental evaluation of the MAPI overhead.

Figures 5 and 6 show some graphic user interfaces (GUIs) that the Manager offers administrators to show service/system conditions. In particular, Figure 5 reports the monitoring data about four Java threads in execution on three hosts of its target domain A. Apart from thread names and execution hosts, the GUIs report monitoring information about thread priority, allocated memory/objects, loaded classes, contended monitors, invoked methods, in/out TCP/UDP packets and lifetime.

Figure 6 visualizes some MAPI indicators about running processes and network traffic on two Windows NT monitored targets. For any process, the GUIs show the process name and identifier, the current CPU usage percentage, the physical/virtual allocated memory, the composing threads and the detailed CPU usage. Network traffic information includes the total number of sent/received TCP segments and UDP/IP packets. Authorized system/service administrators can choose between different forms of visualization of monitoring data, such as the pie charts and histograms shown in the figures.

\section{MONITORING AND CONTROL OF MOBILE AGENT RESOURCE CONSUMPTION}

The SOMA platform itself uses MAPI to monitor and limit mobile agent operations at runtime and, consequently, to control the consumption of distributed resources in SOMAbased Web services. The ultimate goal is to charge the responsible principals for the actions of their mobile agents and to prevent denial-of-service attacks by either malicious or badly programmed agents.

SOMA administrators can define agent permissions and duties, which DM controls and enforces dynamically. Agent permissions specify the actions agents are authorized to perform on a set of resources, even depending on runtime conditions. Agent duties specify the actions agents must perform on a set of target resources when a specified condition takes place [19].

As an example of agent permission specification, one administrator can control the disk resources consumed in a target domain with a distributed authorization policy that limits the total number of bytes that a specified SOMA agent can write during one day over the target hosts in the domain. This limit can also apply to groups of agents, e.g. the whole set of agents of the same responsible principal. At any request of file opening in writing mode by one of the specified agents, the Manager commands one Explorer to probe the target domain. This introduces delay in agent authorizations but permits global policies that depend on the current distributed state. Let us point out that, even in this simple example, the Explorer significantly benefits from the possibility of bringing its reached execution state with it while migrating in the target domain.

As an example of agent duty specification, one administrator can request an Explorer agent to lower autonomously the priority of a SOMA agent running on a host when the total CPU usage percentage on that host is higher than a specified threshold. Differently from the case of permissions, the Manager commands the Explorer to control the state of the CPU usage on the specified host periodically, depending on the interval time indicated in the duty specification. When the threshold is overcome, the Explorer itself acts on the specified agent by exploiting MAPI functions to modify dynamically the Java thread priority.

The conditions for SOMA agent permissions/duties can also be expressed as complex functions of MAPI monitoring indicators. The above examples show that it is often necessary not only to monitor the application state of SOMA agents and of related Java threads, but also to combine these data with kernel and application state of service components even external to the JVM.

\section{MAPI PERFORMANCE}

On-line monitoring tools should limit introduced overhead since they should operate during service provision. We have carefully followed the guidelines of overhead limitation, together with the feature of dynamic tuning, in the design and implementation of DM. To validate MAPI applicability, we have measured the overhead on different platforms, e.g. Intel Pentium III $600 \mathrm{MHz}$ PCs with either Microsoft Windows NT 4.0 or SuSE Linux 6.2, and SUN Ultra 5 $400 \mathrm{MHz}$ workstations with Solaris 7. The hosts are interconnected via $10 \mathrm{Mb}$ Ethernet Local Area Networks (LANs).

This section reports first the costs of local MAPI Profiler Agent and MAPI*ResManager modules. For the evaluation of their performance results, we have used a Java benchmark application that stresses CPU and memory usage by generating a fixed number of different threads and objects. In particular, we report the measurements for the case of 50 benchmark processes in execution, each one with an average number of five threads. We have measured the time delay due to the monitoring tool intrusion as the overhead percentage (Overhead\%), i.e. the difference of $T_{\text {Mon }}$ and $T_{\text {noMon }}$ (normalized to $T_{\text {noMon }}$ ):

$$
\text { Overhead } \%=\left(\frac{T_{\text {Mon }}-T_{\text {noMon }}}{T_{\text {noMon }}}\right) \times 100
$$

where $T_{\text {Mon }}$ is the average completion time of the Java benchmark on unloaded targets with the MAPI tool in execution and $T_{\text {noMon }}$ is the analogous time measured for the benchmark without MAPI. Let us note that unloaded targets represent the worst possible case for the Overhead\% indicator. In fact, as soon as the load increases, $T_{\text {noMon }}$ grows faster than $T_{\mathrm{Mon}}$, and, consequently, the ratio of their difference tends to decrease. This consideration has been validated by measurements of Overhead\% with several general-purpose benchmark tests running: the measurements have shown an average decrease of about $1.0-1.5 \%$ of the Overhead\% indicator for average load conditions. 


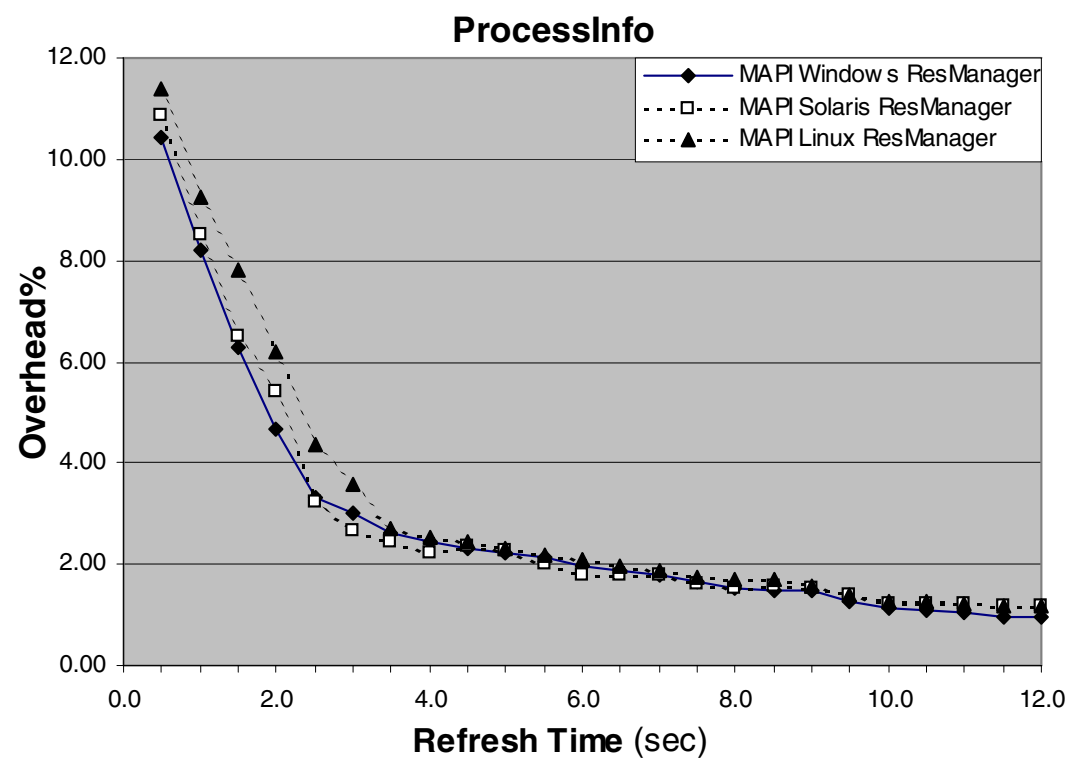

FIGURE 7. Overhead\% of MAPI*ResManagers for process monitoring.

Figure 7 depicts the Overhead\% introduced by the MAPI*ResManager to monitor the whole information contained in Processinfo for the three platforms. The graph reports Overhead\% as a function of the refresh time interval, i.e. the interval between two successive invocations of the native monitoring modules via JNI. Overhead\% exhibits a linear dependence with the reciprocal of the refresh time. We have obtained the same trends in intrusion measurements for native monitoring of NetworkInfo and FilesystemInfo.

In general, the whole set of tests shows that the MAPI*ResManager Overhead\% is always lower than $3 \%$ when the refresh time interval is greater than 3 seconds. This is largely acceptable because this refresh time is enough for the time requirements of most Web-based services. Let us recall that native modules keep on collecting monitored events, and the refresh interval represents only the polling period between successive requests for native monitoring results.

We have also measured the overhead due to the MAPI Profiler Agent. In this case, the JVM notifies events continuously (and not only at specified polling intervals), and the refresh time represents the interval to process the collection of observed events to obtain the concise MAPI indicators. Figure 8 splits the Overhead\% into the parts due to data access and to the JVMPI notification mechanisms, i.e. monitor, method and object tracing. The results obtained for the Solaris platform are very similar to those for Windows NT and Linux, with deviations of the maximum Overhead\% of less than 3\% from the maximum Overhead\%.

Figure 8 also shows that the JVMPI notification is scarcely intrusive under different load conditions and is independent of refresh times. The refresh time seems to affect only the overhead for processing collected events and for reading MAPI indicators. Object tracing has been shown to be the most relevant factor in the MAPI Profiler
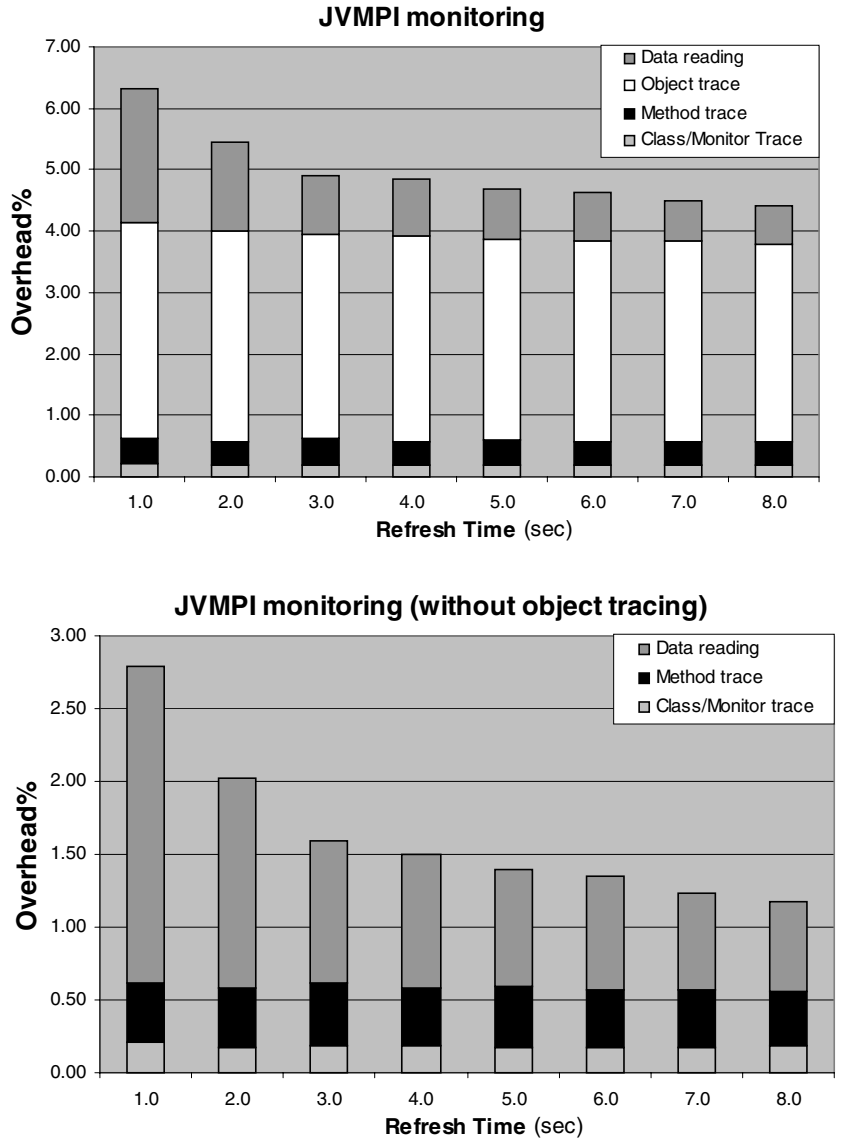

FIGURE 8. Overhead\% of the MAPI Profiler Agent.

Agent intrusion because it requires the profiler to receive and collect a large amount of data. In any case, the total overhead is always below $2.0 \%$, for refresh intervals greater than 2 seconds and with object tracing disabled. 
MAPI Explorer vs. SNMP Client/Server

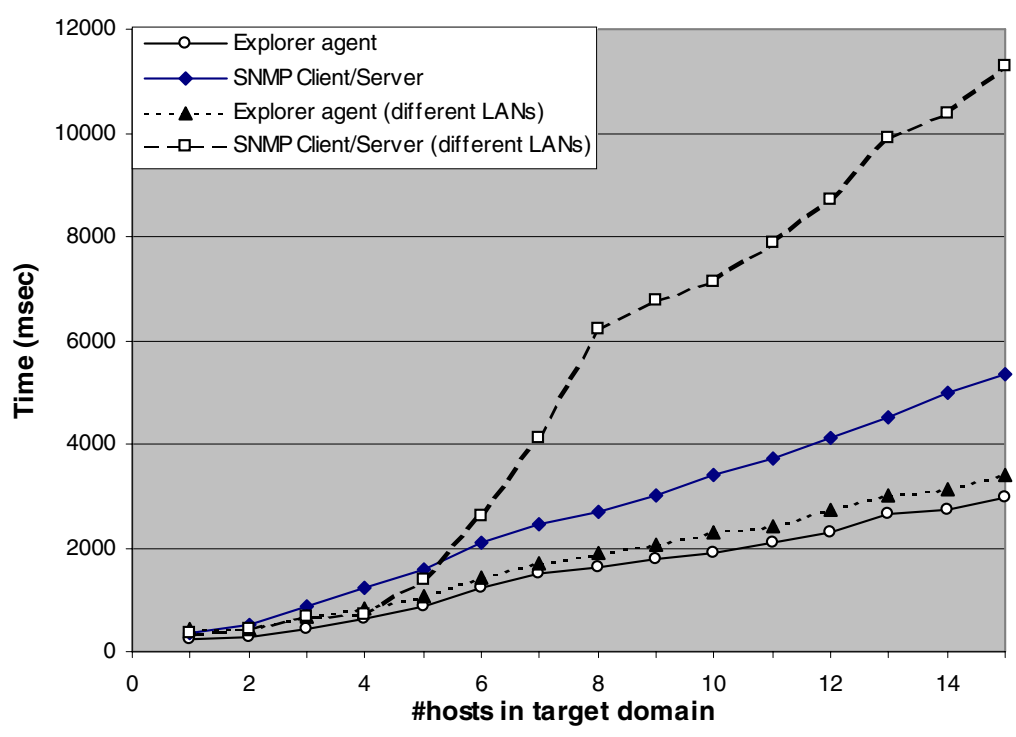

FIGURE 9. MAPI Explorer versus SNMP client/server to monitor one target domain.

Finally, we have also validated the applicability of our mobile-agent-based DM. The Explorer size varies from about $8 \mathrm{kB}$ (at their first migration, without carrying any monitoring indicator) to $15 \mathrm{kB}$ (at the end of exploration, including all the monitoring state of the target domain). Let us note that Explorers need to transfer their code only at the first migration because the SOMA platform at the destination host caches Java classes and triggers the code migration only when it is not available locally [11]. Figure 9 reports the time an Explorer takes to collect monitoring indicators by using MAPI SNMP Agents, depending on the number of hosts in the target domain. The average time has been measured over a large set of experiments, by assuming a 30\% probability of finding the needed agent classes already cached at the destination targets.

The presence of MAPI SNMP Agent permits the collected monitoring data to be filtered and pre-processed. For several different management policies, we have experienced that the MAPI SNMP Agent operations significantly reduce the size of MAPI data collected by the Explorer with respect to the size of corresponding raw MIB values by a factor of three. This optimization is impossible in a traditional client/server approach where a centralized manager should remotely interrogate involved SNMP agents (SNMP client/server graph in the figure). The same experiments confirm that the MA technology is particularly suitable when the target domain includes different LANs, interconnected by low bandwidth links (in our measurements, two Ethernet LANs with $\mathrm{n} / 2$ hosts, connected via a $56 \mathrm{~kb}$ modem link). In this case, Explorer uses the slow link only once to migrate from one LAN to the other. The client/server solution, instead, should operate over the link at least for $n / 2$ SNMP requests and $n / 2$ SNMP replies, wherever the centralized manager is located.

All these results show that, when data filtering is possible and heterogeneity is present, the adoption of mobile agents in DM reduces both time performance and generated traffic. Our results confirm model estimations and first experimental measurements of research work on SNMP distributed monitoring based on mobile agents [13, 21]. In addition, the results point out that the overhead of MAPI*ResManager is significantly higher than that of MAPI SNMP Agent. This has brought us to prefer the latter module when it is available at the target host and supports the Host Resources MIB extensions.

\section{RELATED WORK}

Recent research activities have worked on the definition of methodologies, on the design of architectures and on the implementation of supports for local and distributed monitoring by following very different approaches $[4,7,22,23,24,25,26,27,28,29,30,31,32,33]$.

Several distributed monitoring systems that are based on code instrumentation have achieved interesting results, especially in limiting local overhead and network traffic $[23,26,28]$. However, they require instrumenting either the source code or the binary of managed service components, and force one to recompile or at least restart the monitored services. In addition, the solutions that require source/binary rewriting tend to be language- and platform-specific. This is the main reason why instrumentation-based tools are not a viable solution for monitoring legacy heterogeneous systems and service components in the open and global Internet.

Other research efforts have specifically addressed online monitoring. They typically concentrate on tools that minimize monitoring intrusion, by exploiting ad hoc mechanisms available only for specific operating systems [30, 31]. These solutions also suffer from platform dependency and lack of portability. 
In the area of network monitoring and management, many researchers have focused on standard protocols to exchange information about the state of network equipment. The widespread protocol is still SNMP, mainly because of its simplicity [25]. Other approaches start to be widely employed: some provide network traffic monitoring with the granularity of a whole network segment (Remote MONitoring, RMON [7]); others exploit platformdependent libraries and commands (such as the UNIX libpcap library) to capture, filter and analyze network packets at general-purpose hosts $[34,35]$. These tools, however, aim at the dynamic observation of network traffic and not at making available application-level monitoring data on the state of distributed service components.

The wide adoption of Java in Internet applications has changed the perspective in the monitoring area, by calling for Java also in the implementation of monitoring/management systems. The first activities simply addressed the enhancement of standard SNMP solutions with Web accessibility. Some other proposals started to exploit Java networking facilities and code mobility to provide an integrated middleware for distributed monitoring [36]. The SUN research work in the Java Management API and in the Java Dynamic Management Kit has recently produced the specification and the reference implementation of the Java Management Extensions (JMX) [32]. JMX represents a standard and state-of-the-art solution to instrument monitored resources and to develop management agents. JMX provides an interesting and rich architecture for distributed on-line monitoring, but imposes the instrumentation of the source code of managed resources in accordance with the JMX Instrumentation Level Specification. In addition, monitoring targets should be designed in Java (or enclosed by a Java wrapper). In one sentence, JMX suffers from the same limitations pointed out for instrumentation-based monitoring tools.

Due to the novelty of the technology, there are few examples of Java monitoring tools based on JVMPI. PerfAnal [9] exploits the SUN HPROF profiler agent to perform an off-line analysis of collected monitoring data and to obtain a user-level concise view for debugging Java applications. JProf [8] implements its own profiler agent and process. In addition, it provides a large set of functions to present the off-line data in user-level interoperable formats, such as tables and diagrams organized by using XML. None of the two implements an ad hoc profiler agent for on-line monitoring. In addition, to the best of our knowledge, there are no implemented Java-based tools that currently exploit JNI to integrate with native monitoring mechanisms.

The mobile agent technology has been considered extremely suitable for monitoring and managing distributed systems/services [37]. Several research experiences have demonstrated the mobile agent flexibility and effectiveness to decentralize and to automate the control of service components [13, 18]. However, DM is the first mobileagent-based tool that provides distributed on-line monitoring of both kernel and application states without imposing any modification to the standard JVM and without requiring the instrumentation of the target source/binary code. Both the above requirements are crucial to the application of the tool in legacy systems and service components [33, 38, 39].

\section{CONCLUSIONS AND FUTURE WORK}

The control and management of Web services at runtime require the availability of portable on-line monitoring tools to ascertain the current state of distributed systems and services, without imposing any intervention on the implementation of the monitored targets. These monitoring tools should be core components of any integrated infrastructure for the support of differentiated levels of service quality over best-effort networks.

The MAPI solution addresses this scenario and its performance demonstrates the applicability of Java-based solutions for the on-line monitoring of most classes of Web services. By using MAPI, service developers can tune dynamically the level of detail of monitored events and their refresh time, to adapt the collection of monitoring data (and the corresponding monitoring overhead) to runtime conditions and service-specific constraints. In addition, the mobile agent technology has been shown to be extremely suitable for reducing both monitoring traffic and control latency, by permitting one to filter/process data and to perform management operations directly at the monitored targets.

Given the encouraging performance results achieved, we are currently working on extending the functions and the usability of MAPI-based distributed monitoring. We have extended the MAPI local component to support monitoringbased user accounting in a non-repudiable way, and we have experimented with it in the context of user/terminal mobility [40]. In addition, we are testing the first prototype of an enhanced version of the MAPI-based distributed tool that automatically organizes hierarchies of Managers depending on the topology of the administered systems and service components, thus improving the scalability of our solution when applied to large-scale deployment scenarios. Finally, we are working to increase the usability of the MAPI-based solution by integrating it with user-friendly GUIs for the definition of monitoring/management policies and for their automatic translation in the Ponder Policy Specification Language [19].

\section{REFERENCES}

[1] Lewis, T. (1998) Information appliances: gadget Netopia. IEEE Computer, 31, 59-60.

[2] Chalmers, D. and Sloman, M. (1999) A survey of quality of service in mobile computing environments. IEEE Commun. Surveys Tutorials, 2, 2-10.

[3] Xipeng, X. and Ni, L. M. (1999) Internet QoS: a big picture. IEEE Network, 13, 8-18.

[4] Buyya, R. (2000) PARMON: a portable and scalable monitoring system for clusters. Softw.-Pract. Exper, 30, 723-739.

[5] Sun Microsystems Java Virtual Machine Profiler Interface (JVMPI). http://java.sun.com/products/jdk/1.3/docs/guide/ jvmpi/jvmpi.html. 
[6] Gordon, R. (1998) Essential Java Native Interface. PrenticeHall, Upper Saddle River, NJ.

[7] Stallings, W. (1998) SNMP, SNMPv2, SNMPv3, and RMON 1 and 2 (3rd edn). Addison-Wesley, Reading, MA.

[8] Pennington, G. and Watson, R. (2000) JProf-a JVMPI Based Profiler. http://starship.python.net/crew/garyp/jProf.html.

[9] Meyers, N. (2001) PerfAnal: a Performance Analysis Tool. http://developer.java.sun.com/developer/technicalArticles/ GUI/perfanal/index.html.

[10] Bellavista, P., Corradi, A. and Stefanelli, C. (2000) An integrated management environment for network resources and services. IEEE J. Selected Areas Commun. (Special Issue on Recent Advances in Network Management and Operations), 18, 676-685.

[11] Bellavista, P., Corradi, A. and Stefanelli, C. (2000) Protection and interoperability for mobile agents: a secure and open programming environment. IEICE Trans. Commun. (IEICE/IEEE Special Issue on Autonomous Decentralized Systems), E83-B, 961-972.

[12] Waldbusser, S. and Grillo, P. (2000) Host Resources MIB, RFC2790. http://www.ietf.org/rfc/.

[13] Gavalas, D., Ghanbari, M., O'Mahony, M. and Greenwood, D. (2000) Enabling mobile agent technology for intelligent bulk management data filtering. In Proc. of NOMS'00, Honolulu, HI, April 11-13, pp. 865-876. IEEE Press, Piscataway, NJ.

[14] Entrust Technologies. Entrust/PKI. http://www.entrust.com/products/pki/.

[15] Nemeth, E., Snyder, G., Hein, T. R. and Seebass, S. (2000) UNIX System Administration Handbook (3rd edn). PrenticeHall, Upper Saddle River, NJ.

[16] Bellavista, P., Corradi, A. and Stefanelli, C. (2000) A mobile agent infrastructure for terminal, user and resource mobility. In Proc. of NOMS'00, Honolulu, HI, April 11-13, pp. 877890. IEEE Press, Piscataway, NJ.

[17] Goldszmidt, G. and Yemini, Y. (1995) Distributed management by delegation. In Proc. of ICDCS'95, L'Aquila, Italy. IEEE Computer Society, Los Alamitos, CA.

[18] Pagurek, B., Wang, Y. and White, T. (2000) Integration of mobile agents with SNMP: why and how. In Proc. of NOMS'O0, Honolulu, HI, April 11-13, pp. 609-622. IEEE Press, Piscataway, NJ.

[19] Corradi, A., Dulay, N., Montanari, R. and Stefanelli, C. (2001) Policy-driven management of agent systems. In Proc. of the Policy Workshop, Bristol, January 12-14, pp. 214-229, Springer, Berlin.

[20] Java Community Process. Application Isolation API Specification (Java Specification Request \#121). http://www.jcp.org/jsr/detail/121.jsp.

[21] Baldi, M. and Picco, G. P. (1998) Evaluating the tradeoffs of mobile code design paradigms in network management applications. In Proc. of ICSE'98, pp. 146-155. IEEE Computer Society, Los Alamitos, CA.

[22] Al-Shaer, E., Abdel-Wahab, H. and Maly, K. (1999) HiFi: a new monitoring architecture for distributed systems management. In Proc. of ICDCS'99, pp. 171-178. IEEE Computer Society, Los Alamitos, CA.

[23] Bakic, A., Mutka, M. W. and Rover, D. T. (2000) BRISK: a portable and flexible distributed instrumentation system. Softw.-Pract. Exper., 30, 1353-1373.

[24] Corradi, A. and Stefanelli, C. (1997) HOLMES: a tool for monitoring heterogeneous architectures. In Proc. of HPC'97, pp. 486-491. IEEE Computer Society, Los Alamitos, CA.

[25] Jiao, J., Naqvi, S., Raz, D. and Sugla, B. (2000) Toward efficient monitoring. IEEE J. Selected Areas Commun., 18, 723-732.

[26] Lange, F., Kroeger, R. and Gergeleit, M. (1992) JEWEL: design and implementation of a distributed measurement system. IEEE Trans. Parallel Distrib. Syst., 3, 657-671.

[27] Liang, Z., Sun, Y. and Wang, C. (1999) ClusterProbe: an open, flexible and scalable cluster monitoring tool. In Proc. of IWCC'99, Melbourne, Australia, December 2-3, pp. 57-64. IEEE Computer Society, Los Alamitos, CA.

[28] Miller, B. P., et al. (1995) The Paradyn parallel performance measurement tools. IEEE Computer, 28, 37-46.

[29] Russ, S. H., et al. (1999) Hector: an agent-based architecture for dynamic resource management. IEEE Concurrency, 7, $47-55$.

[30] Schroeder, B. A. (1995) On-line monitoring: a tutorial. IEEE Computer, 28, 72-78.

[31] Weiming, G., Eisenhauer, G., Schwan, K. and Vetter, J. (1998) Falcon: on-line monitoring for steering parallel programs. Concurr-Pract. Exper., 10, 699-736.

[32] SUN Microsystems. Java Management Extensions (JMX). http://java.sun.com/products/JavaManagement/.

[33] Guiagoussou, M. H., Boutaba, R. and Kadoch, M. (2001) A Java API for advanced faults management. In Proc. of IM'01, Seattle, WA, May 14-18, pp. 657-665. IEEE Press, Piscataway, NJ.

[34] Deri, L. and Suin, S. (2000) Effective traffic measurement using Ntop. IEEE Commun. Mag., 38, 138-143.

[35] Kumar, A. et al. (2000) Nonintrusive TCP connection admission control for bandwidth management of an internet access link. IEEE Commun. Mag., 38, 160-167.

[36] Lee, J. (2000) Enabling network management using Java technologies. IEEE Commun. Mag., 38, 116-123.

[37] Fuggetta, A., Picco, G. P. and Vigna, G. (1998) Understanding code mobility. IEEE Trans. Softw. Eng., 24, 342-361.

[38] Czajkowski, G. and von Eicken, T. (1998) JRes: a resource accounting interface for Java. In Proc. of OOPSLA'98, Vancouver, Canada, October 18-22, pp. 21-35. ACM Press, Broadway, NY.

[39] Suri, N., Bradshaw, J. M., Breedy, M. R., Groth, P. T., Hill, G. A. and Jeffers, R. (2000) Strong mobility and fine-grained resource control in NOMADS. In Proc. of ASA/MA'00, Zurich, Switzerland, September 13-15, pp. 2-15. Springer, Berlin.

[40] Bellavista, P., Corradi, A. and Vecchi, S. (2002) Mobile agent solutions for accounting management in mobile computing. In Proc. of ISCC'02, Taormina, Italy, July 1-4, pp. 753-760. IEEE Computer Society Press, Los Alamitos, CA. 